\section{ORIGINAL RESEARCH}

Y. Adachi

A. Poduri

A. Kawaguch

G. Yoon

M.A. Salih

F. Yamashita

C.A. Walsh

A.J. Barkovich

\title{
Congenital Microcephaly with a Simplified Gyral Pattern: Associated Findings and Their Significance
}

BACKGROUND AND PURPOSE: Primary microcephaly is an incompletely understood malformation that is often associated with developmental brain anomalies, yet whether the associated anomalies result from the microcephaly itself or from associated developmental/genetic mishaps is not yet understood. This study reviewed and analyzed a large number of MR imaging scans of children with microcephaly to determine the frequency of associated morphologic findings and to assess whether these findings were associated with the severity of the microcephaly

MATERIALS AND METHODS: MR images of 119 patients with clinically diagnosed microcephaly were retrospectively reviewed, focusing on the degree of microcephaly, simplification of gyri, white matter volume, abnormalities of the corpus callosum, size and structure of posterior fossa contents, and myelination. Associations among the findings were evaluated by using the Spearman correlation coefficient and the Fisher exact test.

RESULTS: Among 7 patients with mild, 42 with moderate, and 70 with extreme microcephaly, a significant correlation was identified between a greater degree of microcephaly and both a greater degree of simplified gyration and decreased white matter volume. The severity of the callosal anomaly showed a lower but still significant correlation with the severity of microcephaly. Degree of hypoplasia of posterior fossa structures, delay in myelination, and abnormality of the basal ganglia did not correlate with the degree of microcephaly.

CONCLUSIONS: A strong correlation was found between the degree of microcephaly, the volume of white matter, and the presence of a simplified gyral pattern. These associations should be considered when attempting to use neuroimaging for segregation and classification of patients with microcephaly.

ABBREVIATIONS: $B G=$ basal ganglia; $M S G=$ microcephaly with simplified gyral pattern; $W M=$ white matter

C ongenital microcephaly (a very small head at birth) can be divided into 2 major categories: primary and secondary (acquired). Patients with primary congenital microcephaly have been described as having congenitally small but architecturally normal brains (thus distinguishing them from microcephaly associated with cerebral cortical malformations, such as holoprosencephaly or lissencephaly), typically associated with nonprogressive mild mental retardation. ${ }^{1,2}$ The cause has been presumed to be genetic. In contrast, acquired micro-

Received July 11, 2010; accepted after revision October 19

From the Department of Radiology and Biomedical Imaging (Y.A., A.J.B.), University of California, San Francisco, San Francisco, California; Division of Genetics (A.P., C.A.W.), Manton Center for Orphan Disease Research and the Howard Hughes Medical Institute, Children's Hospital Boston, Boston, Massachusetts; Biostatistics Center (A.K.), Kurume University, Fukuoka, Japan; Divisions of Clinical/Metabolic Genetics and Neurology (G.Y.), The Hospital for Sick Children, Toronto, Ontario, Canada; Division of Pediatric Neurology (M.A.S.), College of Medicine and King Khalid University Hospital, King Saud University, Riyadh, Saudi Arabia; and Department of Radiology (F.Y.), National Center Hospital of Neurology and Psychiatry, Tokyo, Japan.

C.A.W. and A.J.B. are supported by the National Institute of Neurological Disorders and Stroke, grant 2R01NS035129. C.A.W. is an Investigator for the Howard Hughes Medical Institute.

Please address correspondence to Yuko Adachi, MD, Department of Radiology and Biomedical Imaging, University of California, San Francisco, 505 Parnassus Ave, San Francisco, CA, 94143; e-mail: a-yuko@fb4.so-net.ne.jp

Indicates open access to non-subscribers at www.ajnr.org

Indicates article with supplemental on-line table.

DOI 10.3174/ajnr.A2440

cephaly is presumed to result from brain injury, such as that associated with hypoxic-ischemic injury, intracranial infection, or metabolic disease ${ }^{3}$; head size is initially normal but decreases as a result of brain injury. It is known that some primary microcephalies show an abnormally simplified gyral pattern without thickening of the cerebral cortex; this group has been referred to as MSG. ${ }^{4-9}$ Patients with MSG often have various associated developmental brain anomalies, such as corpus callosal hypogenesis and hypoplasia, periventricular nodular heterotopia, and delayed myelination., ${ }^{2,3}$ Several reports of patients with MSG have been published; however, most were case reports or reviews of a small number of patients. ${ }^{6,7}$ Studies of larger numbers of patients are often useful to gain a better understanding of the disorder and the frequen$\mathrm{cy} /$ significance of associated findings. Therefore, we undertook a retrospective study of MR images of 119 patients with clinically diagnosed primary microcephaly in an attempt to understand the significance of the morphologic findings, including the gyral pattern.

\section{Materials and Methods}

\section{Patients}

A search was made for patients identified with microcephaly from the radiology teaching file at the University of California, San Francisco, the personal teaching file of the senior author (acquired during 26 years), and patients ascertained in a study of the genetics of epilepsy. All patients were clinically diagnosed having as microcephaly and 

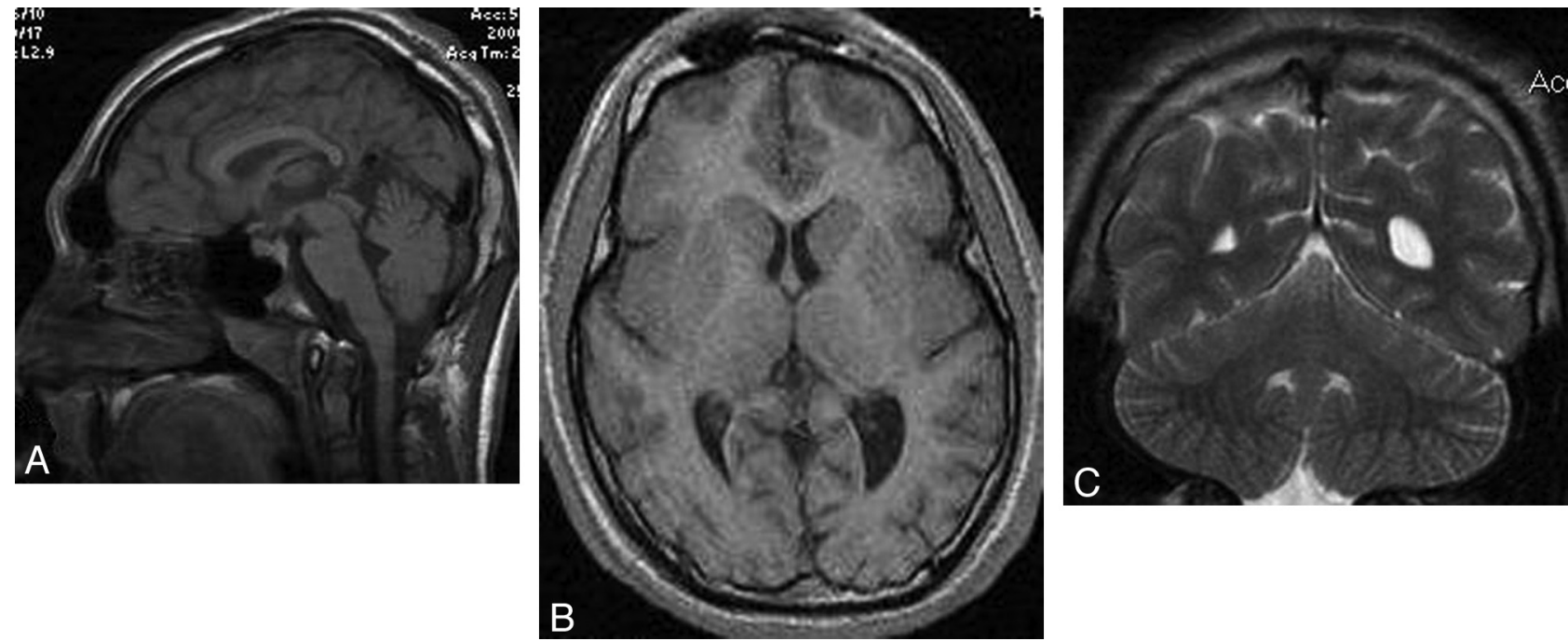

Fig 1. A 14-year-old boy with microcephaly and developmental delay. A, Sagittal T1-weighted image shows extreme microcephaly. The corpus callosum is fully formed but appears diffusely thin. B, Axial T1-weighted image shows a mildly simplified gyral pattern with normal cortical thickness. C, Coronal T2-weighted image shows a disproportionally large cerebellum.
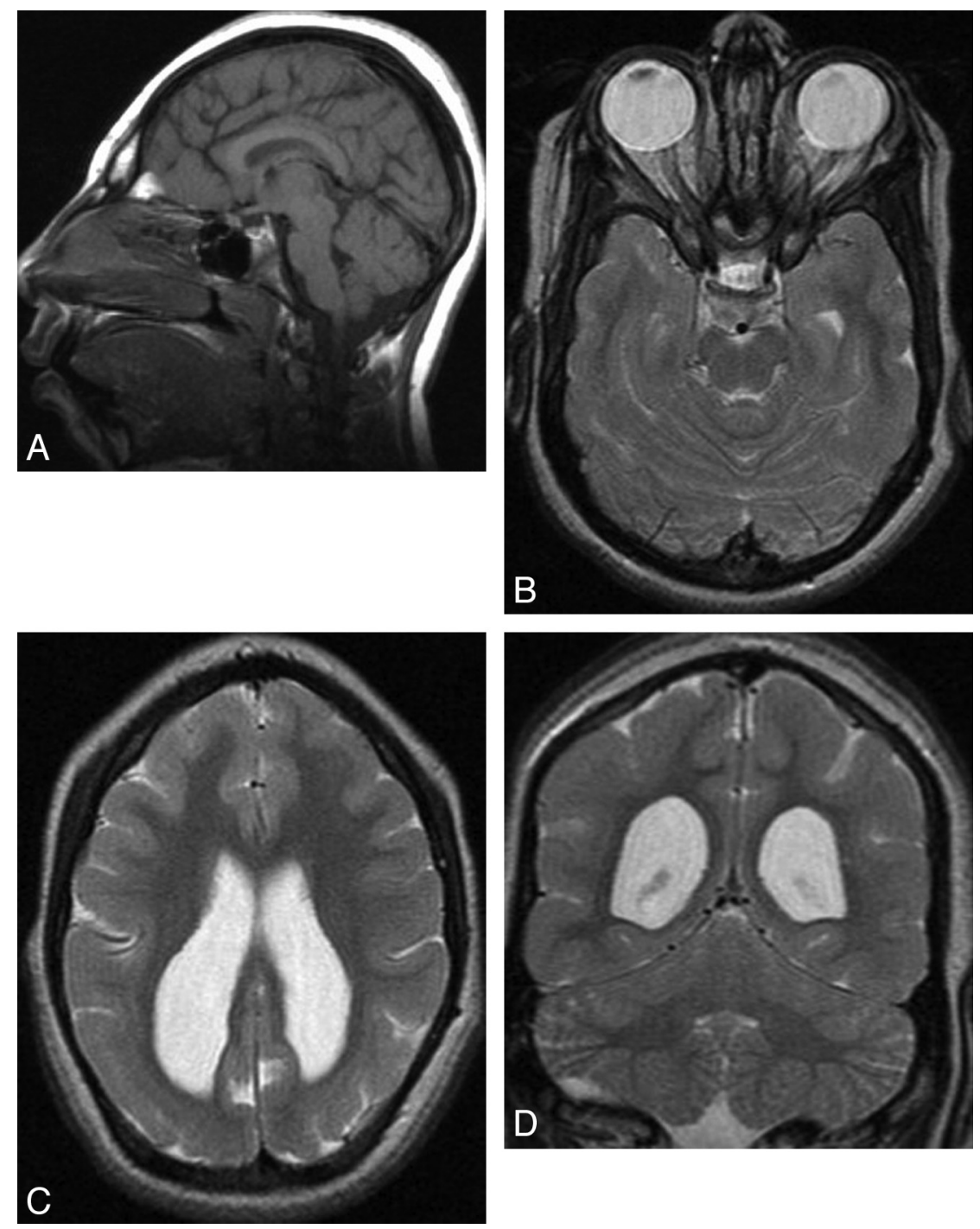

Fig 2. A 10-year-old girl with microcephaly. A, Sagittal T1-weighted image shows extreme microcephaly. The corpus callosum is fully formed but diffusely thin. $B$ and $C, A x i a l$ T2-weighted images show a moderately simplified gyral pattern with too few sulci but normal cortical thickness (measured at $<3 \mathrm{~mm}$ ). Symmetric posterior horn-dominant lateral ventricular enlargement is shown. White matter volume is severely reduced. D, Coronal T2-weighted image shows a disproportionally large cerebellum. 


\begin{tabular}{lcccc}
\hline \multicolumn{5}{l}{ Age distribution of patients with microcephaly } \\
\hline Age & Male & Female & Unknown & Total \\
\hline $0-1$ mo & 11 & 10 & & 21 \\
$1-2$ mo & 7 & 6 & & 13 \\
$3-6$ mo & 4 & 10 & & 14 \\
$7-12$ mo & 6 & 8 & 1 & 14 \\
$13-24$ mo & 9 & 5 & & 15 \\
$2-3$ yr & 7 & 4 & 1 & 11 \\
$3-6$ yr & 7 & 5 & & 12 \\
$6-10 \mathrm{yr}$ & 3 & 7 & 4 & 11 \\
$>10 \mathrm{yr}$ & 2 & 2 & \\
unknown & & & & \\
\hline
\end{tabular}

were referred for imaging after measurements indicated a head circumference $>2$ SDs below the mean head circumference for age during the first 6 months of life. A total of 119 patients with microcephaly were identified. The age distribution of patients is summarized in the Table (56 males and 57 females, ranging in age from 1 day to 30 years old-only 16 patients were older than 6 years; no information about age or sex was available in 4 patients). Eighteen patients were related to other patients in the study, coming from a total of 9 pedigrees. Four patients were born to consanguineous parents (consanguineous: intermarriage among relatives of the third degree or closer). No overlap was found between pedigrees with several affected patients and consanguineous marriages. Evidence of brain damage, resulting from an ischemic or infectious event, metabolic disease, or generalized cerebral malformation (such as diffuse polymicrogyria, lissencephaly, or holoprosencephaly) was a cause for exclusion from this series. A thick cortex (lissencephaly) was a cause for exclusion from the study: The cortex was measured if there was any question of increased cortical thickness. A cortex measuring $\geq 4$-mm thickness eliminated that patient from the study. The clinical and genetic data available on the subjects in the study were highly variable and, in many cases, extremely limited or even nonexistent (other than the diagnosis of microcephaly, which was the reason for examination) because the MR images were obtained from numerous sources in various locations during many years. Therefore, this evaluation focused exclusively upon imaging findings.

Normal comparison data for this study were obtained by assessing craniofacial ratios and white matter volumes in 30 normocephalic infants and children scanned by MR imaging for indications that should not affect head size or white matter volumes. These included healthy neonates ascertained for other studies and infants/children studied for headache, behavioral disorders, ophthalmologic disorders, suspected infection (only included if none was found), and febrile seizures; all were interpreted as studies with normal findings by fellowship-trained neuroradiologists and verified as normal by at least 1 of the authors. These patients ranged in age from 3 months to 5 years at the time of their MR imaging and included 6 neonates, four 3-6 months of age, three 6-12 months of age, four 1-2 years of age, five $2-3$ years of age, and eight $3-5$ years of age. The presence of any brain abnormality on the MR imaging excluded that patient as a control.

\section{MR Imaging Examinations}

Because the MR imaging studies were performed during 20 years at many different imaging centers and on many different types of MR imaging scanners, the imaging techniques that were used differed considerably. All examinations in this study included at least 1 sagittal imaging sequence with section thickness of $\leq 5 \mathrm{~mm}$ and 1 axial imaging sequence with sections of $\leq 5 \mathrm{~mm}$. All patients were studied with both T1- and T2-weighted sequences, and $84.0 \%$ had sequences performed in 3 orthogonal planes. The absence of volumetric datasets in most studies precluded quantification of brain volumes.

\section{MR Imaging Analysis}

Imaging assessment in the patient group was based on agreement between 2 neuroradiologists who reviewed the images. Each neuroradiologist made initial evaluations independently, and any disagreements regarding the final conclusion were resolved by consensus.

Microcephaly. Degree of microcephaly was visually analyzed by using midsagittal T1-weighted images to assess the craniofacial ratio (ratio of the area of the intracranial structures to the area of the face) in the midsagittal plane. The size of the facial area, from the bottom of the chin to the top of the orbit and from the front of the nose to the back of the orbit, was visually assessed on the midline sagittal image and compared with the size of the remainder of the craniofacial area on that same image. The craniofacial ratio is known to be large at birth and to slowly diminish with increasing age; a small craniofacial ratio is considered an indication of microcephaly. ${ }^{10}$ Because the precise head circumference at the time of imaging was not known in many patients, the craniofacial ratios in our subjects were compared with the age-matched normocephalic children described above, and these results were used to classify the severity of microcephaly. We defined "mild microcephaly" as having a craniofacial ratio reduced by $\leq 30 \%$ compared with age-matched normocephalic children; moderate microcephaly was reduced by $30 \%-50 \%$; and extreme microcephaly, by $>50 \%$.

Gyral Pattern. We defined a grading system for sulcation on the basis of the method of van der Knaap et al. ${ }^{11}$ In a normal pattern, gyri and sulci are branched appropriately for age according to published standards. ${ }^{12,13}$ The gyral pattern was considered mildly simplified if the width of the gyri was less than the depth of the sulci, moderately simplified if the width of the gyri was equal to the depth of the sulci, and severely simplified if the width of the gyri was greater than or equal to the depth of the sulci. If the severity of simplification varied in different parts of the cerebrum, we used the grade of the most severe area for evaluation. None of the patients whose scans we studied had myelination patterns compatible with premature birth; therefore, it seems extremely unlikely that immature sulcation was mistaken for simplified sulcation.

White Matter Volume. We evaluated white matter volume separately from the degree of microcephaly. The severity of the reduction of white matter volume was judged mainly by the proximity of the depths of the sulci to the lateral margins of the lateral ventricles. We defined mild loss of white matter volume as the distance from the depths of the sulci to the ventricular wall being reduced by $10 \%-30 \%$ compared with age-matched normocephalic children, moderate loss if the distance was reduced by $30 \%-70 \%$, and severe loss if the distance was reduced by $>70 \%$ compared with age-matched controls. Quantitative volumetric analysis was precluded by the absence of volumetric imaging data in most of our cases.

Other Structures. The corpus callosum, posterior fossa structures, deep gray matter, degree of myelination, and presence of any associated malformations were assessed on each MR image. The corpus callosum was subjectively assessed on midsagittal T1-weighted images and was classified as normal, hypoplastic (fully formed but too thin), hypogenetic (partially formed), or completely absent. The posterior fossa structures (the cerebellum, midbrain, pons, and medulla) were visually assessed for morphology and size; the sizes of the brain stem and cerebellum were compared with those of the cerebrum in each patient to determine 

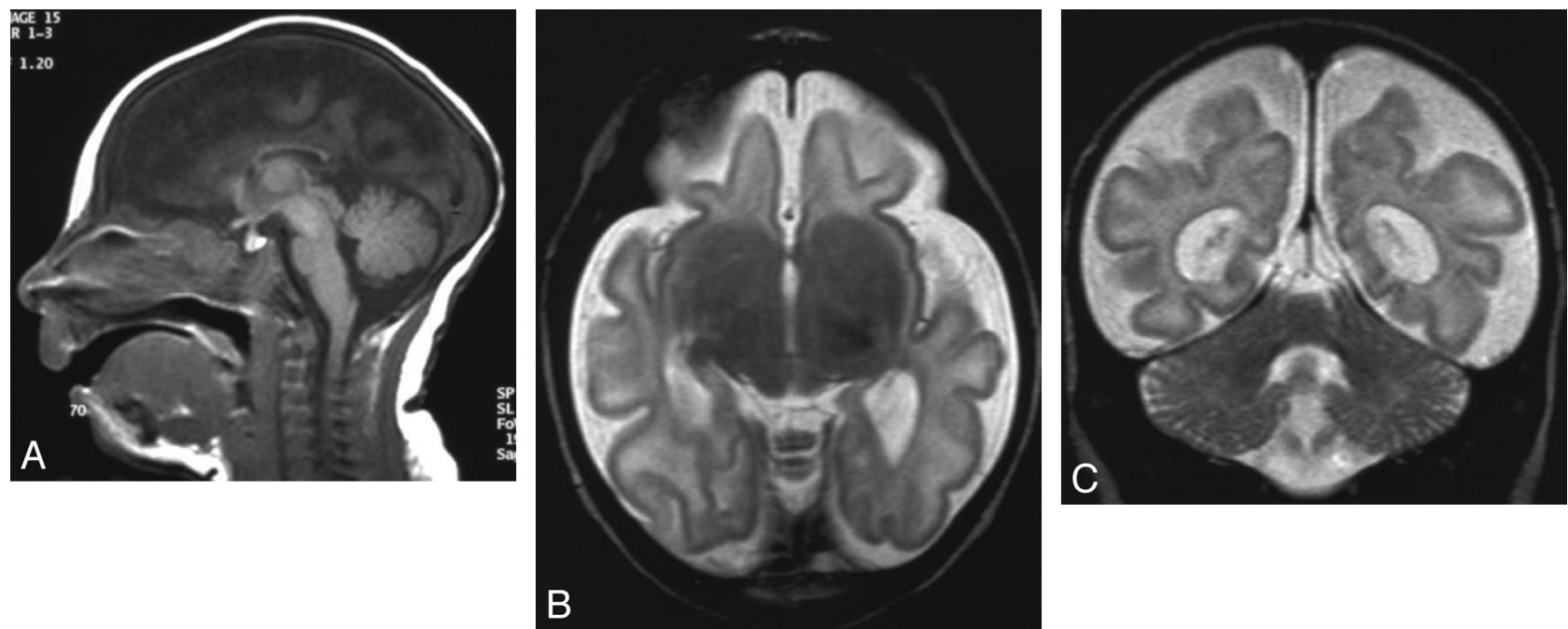

Fig 3. A 1-month-old girl with microcephaly, global developmental delay, and seizures. A, Sagittal T1-weighted image shows extreme microcephaly. The corpus callosum is partially formed the rostrum is absent, and other parts (genu, body, and splenium) are diffusely thin. B, Axial T2-weighted image shows a severely simplified gyral pattern; sulci are both too few and too shallow. Although myelination is shown in the posterior limb of the internal capsule, the abnormal high intensity of the white matter is observed diffusely and the volume of the white matter is severely diminished. $C$, Coronal T2-weighted image shows a disproportionally large cerebellum.
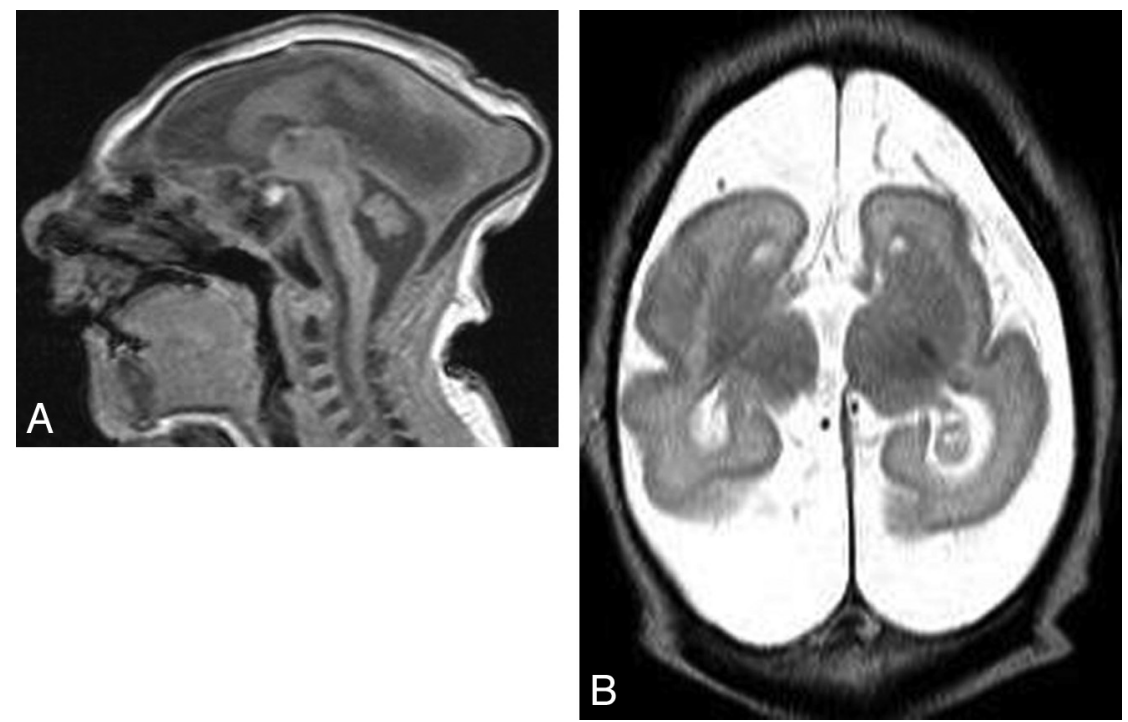

Fig 4. A 2-week-old boy with profound microcephaly. A, Sagittal T1-weighted image shows extreme microcephaly with absence of the corpus callosum. All structures seem proportionately affected. B, Axial T2-weighted image shows a severely simplified gyral pattern (almost no sulci). The volume of white matter is also extremely reduced proportional to the simplification of the gyral pattern, and the pericerebral spaces are enlarged.

whether they were proportional or disproportional (disproportionally large/small) to the cerebrum. The size and configuration of the thalami and basal ganglia were evaluated as normal, small, or dysmorphic. Myelination was assessed on T2-weighted images and compared with published standards ${ }^{12}$ and with age-matched healthy controls. Associated malformations (heterotopias and interhemispheric cyst) were also visually assessed and recorded if present.

Our review was retrospective because the MR imaging examinations were performed during many years at numerous institutions. Therefore, it was impossible to obtain informed consent from all patients or their families. The Committee on Human Research approved this retrospective study at the University of California, San Francisco.

\section{Statistical Analysis}

Correlations of the degree of microcephaly with other structures (gyral pattern, white matter volume, corpus callosum, posterior fossa structures, myelination, and basal ganglia) were assessed by computing the Spearman correlation coefficient and the Fisher test. $P$ values below .05 were considerate significant.

\section{Results}

We identified 7 mild, 42 moderate, and 70 extreme microcephaly cases. Results of the cases are summarized in the On-line Table.

\section{Simplified Gyral Pattern}

In the 7 cases of mild microcephaly, 2 patients had normal sulcation, 4 had a mildly simplified gyral pattern, and 1 had a severely simplified gyral pattern. No patient was assessed as having a moderately simplified gyral pattern. The group of moderate microcephaly was composed of 42 patients. Nine of the patients had normal gyration, 24 had mildly simplified 
gyral patterns, 8 had moderately simplified gyral patterns, and 1 had a severely simplified gyral pattern. The group with extreme microcephaly included 70 cases: Five patients had normal gyration, 18 had mildly simplified gyral patterns (Fig 1), 20 had moderately simplified gyral patterns (Fig 2), and 27 had severely simplified gyral patterns (Fig 3).

\section{White Matter Volume}

All 119 patients with microcephaly had subjectively reduced white matter volume. Using the method defined above, we identified mildly reduced white matter volume in 16 patients, moderate in 52 patients, and severe in 51 patients.

Of 16 patients with mild reduction of white matter volume, 2 had mild microcephaly, 10 had moderate microcephaly, and 4 had extreme microcephaly. Normal gyration was identified in 6 of the 16 patients, 9 had mildly simplified gyral patterns, and 1 had a moderately simplified gyral pattern. No patients with severely simplified gyral patterns were identified in this group.

In 52 patients with moderately reduced white matter volume, 4 had mild microcephaly, 21 had moderate microcephaly, and 27 had severe microcephaly (Fig 1). Normal sulcation was identified in 9 of the 52 patients, 25 had mildly simplified gyral patterns (Fig 1), 12 had moderately simplified gyral patterns, and 6 had severely simplified gyral patterns.

In 51 patients with severely reduced white matter volume, 1 had mild microcephaly, 11 had moderate microcephaly, and 39 had severe microcephaly (Fig 3). Normal sulcation was found in 1 of the 51 patients, 12 had mildly simplified gyral patterns, 15 had moderately simplified gyral patterns, and 23 had severely simplified gyral patterns (Fig 3 ).

\section{Other Structures}

The corpus callosum had a subjectively normal shape and thickness in 28 patients. In 6 patients, the corpus callosum could not be assessed because an adequate midsagittal image was not obtained. The remaining 85 patients had abnormalities of the corpus callosum: Fifty-nine patients had callosal hypoplasia (thin but all parts formed) (Fig 1), 21 had callosal hypogenesis (not all callosal parts formed) (Fig 3), and 5 had complete callosal agenesis (Fig 4).

Both the cerebellum and brain stem were proportional in size to the cerebrum in 45 patients. Fifty-four patients had a disproportionally large cerebellum; in all, the brain stem was proportional to cerebellum (Figs 1, 2, and 3). In 19 patients, the cerebellum was disproportionally small. The brain stem was proportional to the cerebellum in 10 of these 19 patients and proportional to cerebrum in 9 of the 19 patients. One patient with a disproportionally small cerebellum had abnormal superior cerebellar peduncles that resembled a molar tooth malformation. In 1 patient, assessment of the posterior fossa was difficult because we had a limited number of MR images.

Small basal ganglia were observed in 20 patients; 10 of the 20 patients had small thalami. Small thalami with normal basal ganglia were found in 10 patients. All 30 patients with small basal ganglia and/or small thalami showed normal shape and gross anatomic relationships of the structures. Abnormal shape and gross anatomic relationship of basal ganglia and/or thalami were identified in 5 patients.

Seventy-seven patients had myelination appropriate for age, whereas 32 patients had delayed myelination. In 5 patients, myelination could not be assessed because of unknown age or extreme reduction of white matter volume.

Associated malformations included periventricular nodular heterotopia $(n=7)$ and an interhemispheric cyst $(n=4)$. One patient had both heterotopia and an interhemispheric cyst.

\section{Statistical Analysis}

Assessment with the Spearman rank correlation test showed a significant correlation between the degree of microcephaly and the degree of simplified gyration $(r=0.47, P<.0001)$. Both white matter volume $(r=0.36, P<.001)$ and abnormality of the corpus callosum $(r=0.26, P<.05)$ showed a lower but still significant correlation with the degree of microcephaly. The size of cerebellum and brain stem relative to the cerebrum showed a negative correlation with the degree of microcephaly $(r=-0.31, P<.001)$. Myelination and abnormality of the basal ganglia did not show any correlation with the degree of microcephaly. Additionally, we assessed the correlation between volume reduction of white matter and degree of gyral simplification. The degree of volume reduction of the white matter showed a significant correlation with a simplified gyral pattern $(r=0.55, P<.001)$.

\section{Discussion}

The most interesting finding in this study was the strong correlation between the degree of microcephaly and the presence of a simplified gyral pattern. Although several previous communications have reported patients with microcephaly and a simplified gyral pattern, most were case reports or reviews of a small number of patients. ${ }^{7-9}$ By their very nature, such reports lack perspective on the significance of their findings. In this study, MR images of 119 patients with clinically diagnosed microcephaly were retrospectively evaluated in an attempt to understand the significance and relationship, if any, of the associated morphologic findings, including the gyral pattern.

Primary microcephaly was originally described as a congenitally small but architecturally normal brain, with affected patients having nonprogressive mild mental retardation. ${ }^{1,2}$ As the interest in primary microcephaly and its genetic causes has grown, many such patients have been studied with MR imaging and have been found to have abnormalities of the cerebral cortex, characterized by a reduced number of gyri and shallow sulci. This group is referred to as having MSG. ${ }^{3}$ Although it is unclear whether primary microcephaly (with normal sulcation) and MSG are part of a phenotypic continuum, it is clear that microcephaly has heterogeneous clinical, radiologic, and genetic features. ${ }^{5-7}$ Previous reports have suggested that patients with MSG may have more severe mental retardation, neurodevelopmental delay, and associated central nervous system malformations than those with primary microcephaly. ${ }^{5,7,9}$ Although MSG was initially subdivided into 6 groups on the basis of the neonatal clinical features and neuroimaging findings, ${ }^{5,14}$ the authors of those articles had few patients in each group and commented that the classification may change as more patients are described. We believe that the larger num- 
ber of imaging studies evaluated in this study has allowed a somewhat better perspective on the significance of the simplified gyral pattern, which is important for management of affected patients and counseling of their relatives (though, ultimately, counseling may be based more on genetic analyses).

Malformations of cortical development are classified on the basis of the stage at which the developmental process was likely disturbed: cell proliferation/apoptosis, neuronal migration, and cortical organization. ${ }^{15}$ Microcephaly is classified as a disorder of cell proliferation, ${ }^{15}$ suggesting that either the number of cycles of cell proliferation is reduced or, perhaps, apoptotic cell death is increased. Recent studies show that many of the proteins that are mutated in primary microcephalies localize to centrosomes for at least part of the cell cycle as discussed by Thornton and Woods. ${ }^{16}$ They hypothesized that the centrosome is a final integration point for many regulatory pathways affecting prenatal neurogenesis in mammals, supporting the concept of primary microcephaly as a disorder of neuronal proliferation. However, the frequency and significance of a simplified gyral pattern among primary microcephalies were not discussed in any classifications that were found by these authors. In our study, a simplified gyral pattern was detected in 103 of 119 cases of primary microcephaly of various severities. Although these cases presumably had many different genetic causes, an association was found between the severity of microcephaly and the degree of simplification of the gyral pattern. These results suggest a potential cause/effect relationship between the microcephaly and the simplified gyral pattern, possibly as an effect of reduced cell proliferation. A reduced number of neurons and axons could cause both microcephaly and the simplified gyral pattern.

Several hypotheses have been put forward on the processes that underlie the normal gyration of the fetal brain. ${ }^{17-19} \mathrm{Al}-$ though the mechanisms of sulcal formation are not well-understood, a respected hypothesis by Van Essen ${ }^{17}$ suggested that cortical folding is related to tension exerted on the developing cortex by axonal connections. Possible factors influencing the tension are the number of subcortical and corticocortical axonal projections, as well as white matter and intracortical myelination. ${ }^{19}$ We postulate that decreased neuronal proliferation results in fewer cortical neurons and, thus, fewer axons exiting the cortex; this result might cause decreased tension arising from axons and, consequently, fewer and more shallow sulci. Moreover, the degree of volume reduction of cerebral white matter also showed a correlation with a simplified gyral pattern. This result supports the concept that gyral simplification reflects reduced tension on the cortex due to the production of fewer axons.

In a more recent study, Toro and Bornod ${ }^{20}$ analyzed the dynamics of cortical folding by using a finite-element implementation of the morphogenetic model; this study suggested that cortical growth also plays a fundamental role in cortical folding. Because cortical growth is directly related to the number of neurons produced, less cortical growth would be expected to result in less sulcation. This consideration further supports our hypothesis that the simplified gyral pattern is, at least partially, a consequence of the degree of microcephaly (more specifically the amount of cortical growth and axonal production) and not a separate unrelated feature of certain types of primary microcephaly.
The term "simplified gyral pattern" is most often used to describe a reduced number of gyri and shallow sulci with a normal cortical thickness. However, this term can be confusing because other terms are sometimes used to describe malformations with too few gyri and too shallow sulci: oligogyria, lissencephaly, pachygyria, and microlissencephaly. ${ }^{3,14}$ It is useful to define these conditions and their causes because these malformations result from different causes and developmental periods. A simplified gyral pattern is considered to result from reduced cell proliferation or increased apoptosis ${ }^{18}$; it is synonymous with oligogyria. When seen in a mature brain (term-equivalent neonate or older), it is almost always associated with microcephaly and reduced white matter volume; the cortex is of normal thickness.

The term "lissencephaly" is used to describe a cortical malformation caused by disturbed neuronal migration. The affected cortex is agyric, and as a result of the disturbed migration, it is abnormally thick, usually at least $1 \mathrm{~cm}$. "Pachygyria" (also called "incomplete lissencephaly") refers to a lesser degree of disturbed migration of neurons. A few shallow sulci are present, and the gyri are broad. This cortex is thick but less so than in lissencephaly, usually $6-9 \mathrm{~mm}$. The few pathologic studies of simplified gyral patterns that have been published have revealed a normal cerebral cortical thickness and normal lamination or subtle alterations of cortical lamination. ${ }^{3,14,21}$ "Microlissencephaly" is a less well-defined term; indeed, it is defined differently in different publications. ${ }^{5,6,14}$ However, it should be characterized by a thickened cortex and thereby can be distinguished from MSG. We have avoided using the term "microlissencephaly" in this study because it may be confusing and misleading. We suggest that this term be avoided and that microcephaly be considered a characteristic sometimes associated with lissencephaly.

A few previous reviews based on the genetics and etiology of microcephaly have been published, ${ }^{1,22,23}$ and the presence of a simplified gyral pattern in association with microcephaly has been discussed by many authors. ${ }^{21,24}$ However, to our knowledge, no previous reports have demonstrated the significant relationship among the severity of microcephaly, the reduced white matter volume, and diminished sulcation.

Our study also revealed a correlation between the degree of microcephaly and the severity of an associated callosal anomaly. In theory, this may be related to the generation of fewer cortical neurons, resulting in production of fewer callosal axons. ${ }^{25}$ This relationship will be investigated in a future study. Microcephaly is an extremely heterogeneous disorder and is also seen in association with various genetic syndromes, including some with small posterior fossa structures. ${ }^{8,9} \mathrm{Al}$ though our study included 19 patients with disproportionally small posterior fossa structures, statistical analysis showed negative correlation between the degree of microcephaly and the size of posterior fossa structures. This result suggests that the cell proliferation of the cerebrum is independent from that of the cerebellum and brain stem in many types of microcephaly.

One potential weakness of this study is that studies of teaching collections, whether personal or institutional, might be biased by a greater tendency for selecting cases that have obvious abnormalities, such as abnormal sulcation or callosal anomalies, compared with those with normal or nearly nor- 
mal proportions of structures. A prospective study would be superior. However, this potential bias would be much more likely in patients with mild microcephaly rather than the moderate or severe microcephaly that was present in most of these infants. Therefore, we do not believe that this was a significant factor, but we cannot completely exclude it. While balancing the numbers by adding more MR images from patients with mild microcephaly might, in some ways, "balance" the study, patients with mild microcephaly may be, paradoxically, more difficult to find, because patients are rarely referred for imaging because of mild microcephaly unless they have associated encephalopathy or focal neurologic deficits. Thus, even if included in a teaching file, the files would be unlikely to have the information about their head size. Practically speaking, therefore, it would be difficult to prospectively find significant numbers of patients with mild microcephaly for a retrospective study such as this. Thus, although we agree that a prospective study of imaging of patients with microcephaly would be interesting and informative, we believe that our results are valid.

In summary, our review of MR imaging studies of a large number of patients with primary microcephaly confirms that microcephaly is a heterogeneous disorder. However, our data suggest that simplification of the gyral pattern may be a consequence of the severity of the microcephaly rather than exclusively a consequence of the underlying cause. Our data also suggest, though less convincingly, that callosal hypogenesis is related to and may be a consequence of the severity of microcephaly. Therefore, these characteristics may not be very useful for classification of patients with microcephaly. A large prospective study of patients with microcephaly with known causes of primary microcephaly would be useful for evaluating other features seen in this disorder and for determining whether they are useful in differentiating microcephalies of different etiologies.

\section{Conclusions}

MR images of 119 patients with clinically diagnosed microcephaly were reviewed. A strong correlation between the degree of microcephaly, the volume of white matter, and the presence of a simplified gyral pattern was revealed. An association of the severity of microcephaly with the presence of a callosal anomaly was also demonstrated, though less convincingly. These associations should be considered when attempting to use neuroimaging for segregation and classification of patients with microcephaly.

\section{Acknowledgments}

We thank the Microcephaly Collaborative for the contribution of patient data that made this study possible and, specif- ically, Grace Yoon, MD, (Toronto), Mustafa Salih, MD, (Riyadh), and Ann Poduri, MD, (Boston) for contributing figures to the manuscript.

\section{References}

1. Warkany J, Lemire RJ, Cohen MM Jr. Mental Retardation and Congenital Malformations of the Central Nervous System. Chicago: Yearbook; 1981:28-30

2. Berg BO. Developmental disorders of the nervous system. In: Berg BO, ed. Principles of Child Neurology. New York: McGraw-Hill; 1996:665-90

3. Mochida GH, Walsh CA. Molecular genetics of human microcephaly. Curr Opin Neurol 2001;14:151-56

4. Passemard S, Titomanlio L, Elmaleh M, et al. Expanding the clinical and neuroradiologic phenotype of primary microcephaly due to ASPM mutations. Neurology 2009;73:962-69

5. Desir J, Cassart M, Van Bogaert P, et al. Primary microcephaly with ASPM mutation shows simplified cortical gyration with antero-posterior gradient pre- and post-natally. Am J Med Genet A 2008;146A:1439-43

6. Peiffer A, Singh N, Leppert M, et al. Microcephaly with simplified gyral pattern in six related children. Am J Med Genet 1999;84:137-44

7. Rajab A, Mansini MC, Mochida GH, et al. A novel form of lethal microcephaly with simplified gyral pattern and brain stem hypoplasia. Am J Med Genet 2007;143A:2761-67

8. Hashimoto K, Takeuchi Y, Kida Y, et al. Three siblings of fatal infantile encephalopathy with olivopontocerebellar hypoplasia and microcephaly. Brain Dev 1998;20:169-74

9. Barth PG, Blennow G, Lenard HG, et al. The syndrome of autosomal recessive pontocerebellar hypoplasia, microcephaly, and extrapyramidal dyskinesia (pontocerebellar hypoplasia type 2): compiled data from 10 pedigrees. $\mathrm{Neu}$ rology 1995;45:311-17

10. Gooding CA. Radiology of the skull and brain In: Newton TH, Potts DG, eds. The Skull. Vol 1. St. Louis: Mosby; 1971:141-43

11. van der Knaap MS, Wezel-Meijler G, Barth PG, et al. Normal gyration and sulcation in preterm and term neonates: appearance on MR images. Radiology 1996;200:389-96

12. Barkovich AJ. Pediatric Neuroimaging. 4th ed. Philadelphia: Lippincott Williams \& Wilkins; 2005:18-41

13. Childs AM, Ramenghi LA, Cornette L, et al. Cerebral maturation in premature infants: quantitative assessment using MR imaging. AJNR Am J Neuroradiol 2001;22:1577-82

14. Barkovich AJ, Ferriero DM, Barr RM, et al. Microlissencephaly: a heterogeneous malformation of cortical development. Neuropediatrics 1998;29:113-19

15. Barkovich AJ, Kuzniecky RI, Jacson GD, et al. A developmental and genetic classification for malformation of cortical development. Neurology 2005;65:1873-87

16. Thornton GK, Woods CG. Primary microcephaly: do all roads lead to Rome? Trends Genet 2009;25:501-10

17. Van Essen DC. A tension-based theory of morphogenesis and compact wiring in the central nervous system. Nature 1997;385:313-18

18. Hilgetag CC, Barbas H. Developmental mechanics of the primate cerebral cortex. Anat Embryol 2005;210:411-17

19. Fischl B, Rajendran N, Busa E, et al. Cortical folding patterns and predicting cytoarchitecture. Cereb Cortex 2008;18:1973-80

20. Toro R, Bornod Y. A morphogenetic model of the development of cortical convolutions. Cereb Cortex 2005; 15:1900-13

21. Friede RL. Developmental Neuropathology. 2nd ed. Berlin, Germany: SpringerVerlag; 1989:299-301

22. Tarrant A, Garel C, Germanaud D, et al. Microcephaly: a radiological review. Pediatr Radiol 2009;39:772-80

23. Woods CG, Bond J, Enard W. Autosomal recessive primary microcephaly (MCPH): a review of clinical, molecular, and evolutionary findings. Am J Hum Genet 2005;76:717-28

24. Huttenlocher PR. Dendritic development in neocortex of children with mental defect and infantile spasms. Neurology 1974;24:203-10

25. Barkovich AJ, Kjos BO. Normal postnatal development of the corpus callosum as demonstrated by MR imaging. AJNR Am J Neuroradiol 1988;9:487-91 